\title{
Minimally invasive direct coronary artery bypass grafting in a 17-year-old patient with left anterior descending coronary artery compression in follow-up after arterial switch operation
}

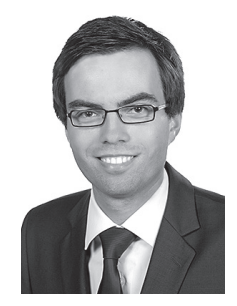

\author{
Andrzej Juraszek, Jarosław Kuriata, Cezary Kępka, Mariusz Kruk, Mariusz Kuśmierczyk \\ Department of Cardiac Surgery and Transplantation, Institute of Cardiology, Warsaw, Poland
}

Kardiochirurgia i Torakochirurgia Polska 2018; 15 (3): 204-206

Transposition of the great arteries is a congenital heart defect of abnormal arrangement of the aorta and pulmonary artery. In the more frequent dextro transposition of the large arteries (d-TGA), the aorta is connected to the right ventricle and the pulmonary artery to the left ventricle of the heart. Therefore, the pulmonary and the body's circulation are separate and the disease is not compatible with life, unless other malformations (for example, an atrial septal defect) are present and the ductus arteriosus Botalli is opened (normally until the first days of life) [1].

The neonatal arterial switch operation is the standard of treatment for transposition of the great arteries. During the procedure the aorta and pulmonary artery are detached from their native roots and reattached to the opposite root. After completion of the operation the pulmonary root is called the neo-aorta, and the aortic root the neo-pulmonary artery. It is necessary to transplant the coronary arteries from the aorta/neo-pulmonary artery to the pulmonary artery/neo-aorta [2].

The peri-operative success rate is about $96 \%$, showing a comparable survival rate after 5 years [3]. One of the drawbacks of this procedure is the risk of late coronary stenosis or occlusion, reported as $11.3 \%$ in a follow-up of 9.6 years [4] or $7.3 \%$ of patients receiving coronary imaging in a meta-analysis of 8798 patients [5]. The first potential manifestation of coronary complications can be sudden cardiac death. To minimize the risk, selective coronary imaging in the follow-up is often performed, despite the low incidence. In the meta-analysis, out of 8798, 27 patients died $\geq 5$ years after the arterial switch operation. Of these patients, 10 had relevant coronary lesions. Five late deaths were sudden, possibly from a cardiac cause [5]. Instead of low sudden cardiac death risk, in patients with significant stenosis surgical revascularization should be considered.

We present a case of a 17-year-old boy with transposition of the great arteries (TGA). He underwent anatomical correction surgery in his first week of life. In the last months he presented signs of myocardial ischemia. Magnetic resonance imaging (MRI) showed ischemia of the anterior left ventricular wall. Cardiac computed tomography (CT, retrospective ECG-gated study with dual source computed tomography Somatom Force, Siemens, Germany using $35 \mathrm{ml}$ contrast medium) and coronary angiography diagnosed compression of the proximal part of the left anterior descending artery (LAD) caused by the neo-aorta and pulmonary trunk (Fig. 1). The local heart team referred the patient for surgery.

The minimally invasive direct coronary artery bypass grafting (MIDCAB) approach was chosen [6]. An anterolateral left thoracotomy in the fifth intercostal space was performed. We found significant pleural adhesions. Than left internal mammary artery (LIMA) was prepared under direct vision, showing a good diameter and flow after the administration of heparin. The LAD was difficult to identify on the cardiac surface due to both the untypical course and previous cardiac adhesions. Finally, the LAD with diameter between 1 and $1.5 \mathrm{~mm}$ was found. Occluding sutures were placed proximally and distally from the planned anastomosis of the LAD and a time of 2 min of ischemia and reperfusion each was chosen for preconditioning. During this time ventricular fibrillation occurred. The occluding sutures were opened and the sinus rhythm was successfully established after defibrillation. Than we decided to start femoral extracorporeal circulation via a cannulas placed in the right femoral artery and vein. Under this support, the ligating sutures were closed, and the patient remained stable. After incision, the LAD anastomosis was performed with a 8/0 running suture with coronary shunt. The extracorporeal circulation was terminated and protamine was administered. After hemostasis the thoracotomy access and the femoral wound were closed.

The recovery was uneventful. The patient required no catecholamine support. He was extubated on the first postoperative day and discharged home after 6 days. Postoperative CT showed adequate blood flow in the coronary bypass (Fig. 2). 

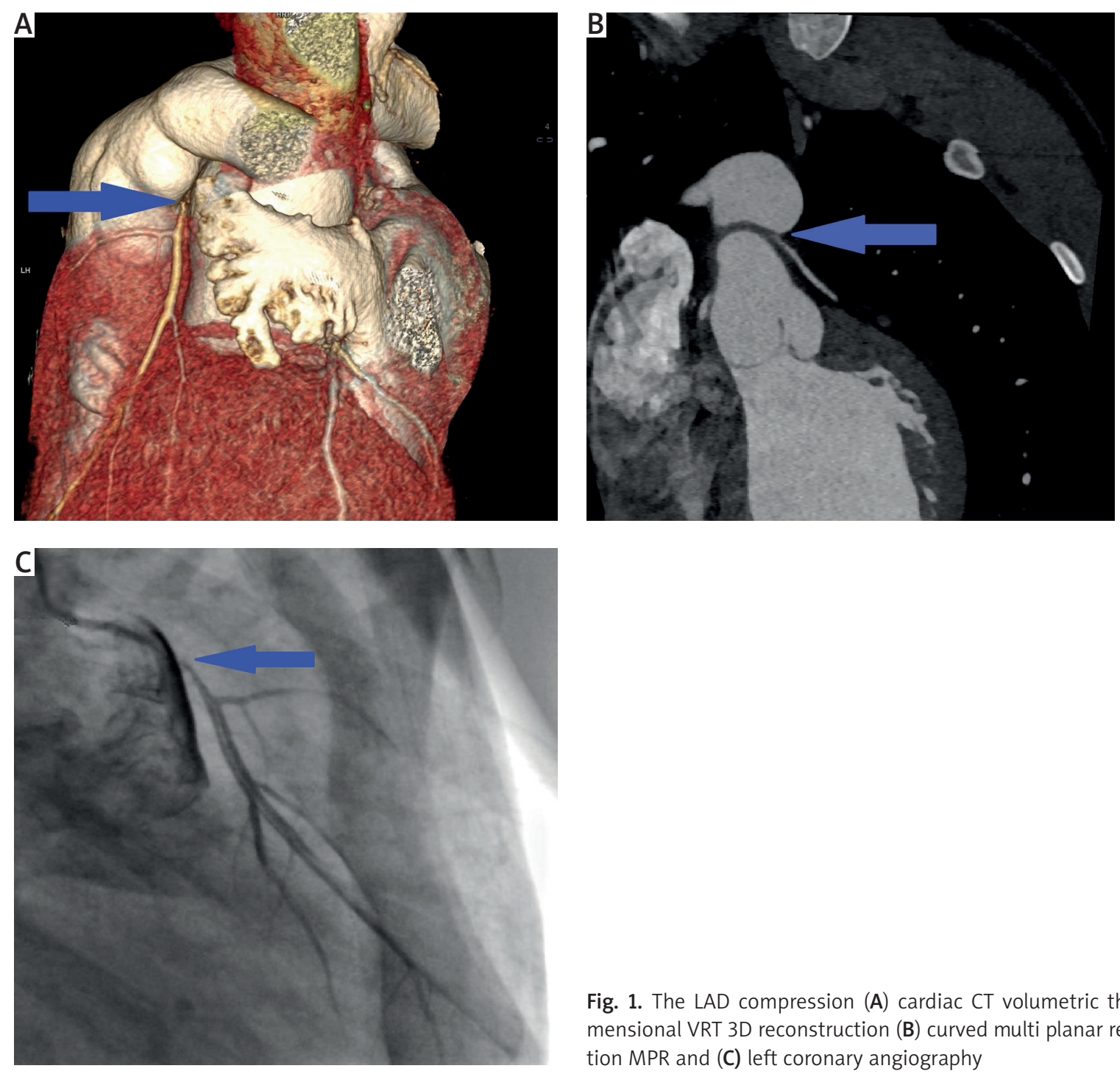

Fig. 1. The $L A D$ compression (A) cardiac CT volumetric three dimensional VRT 3D reconstruction (B) curved multi planar reformation MPR and $(C)$ left coronary angiography
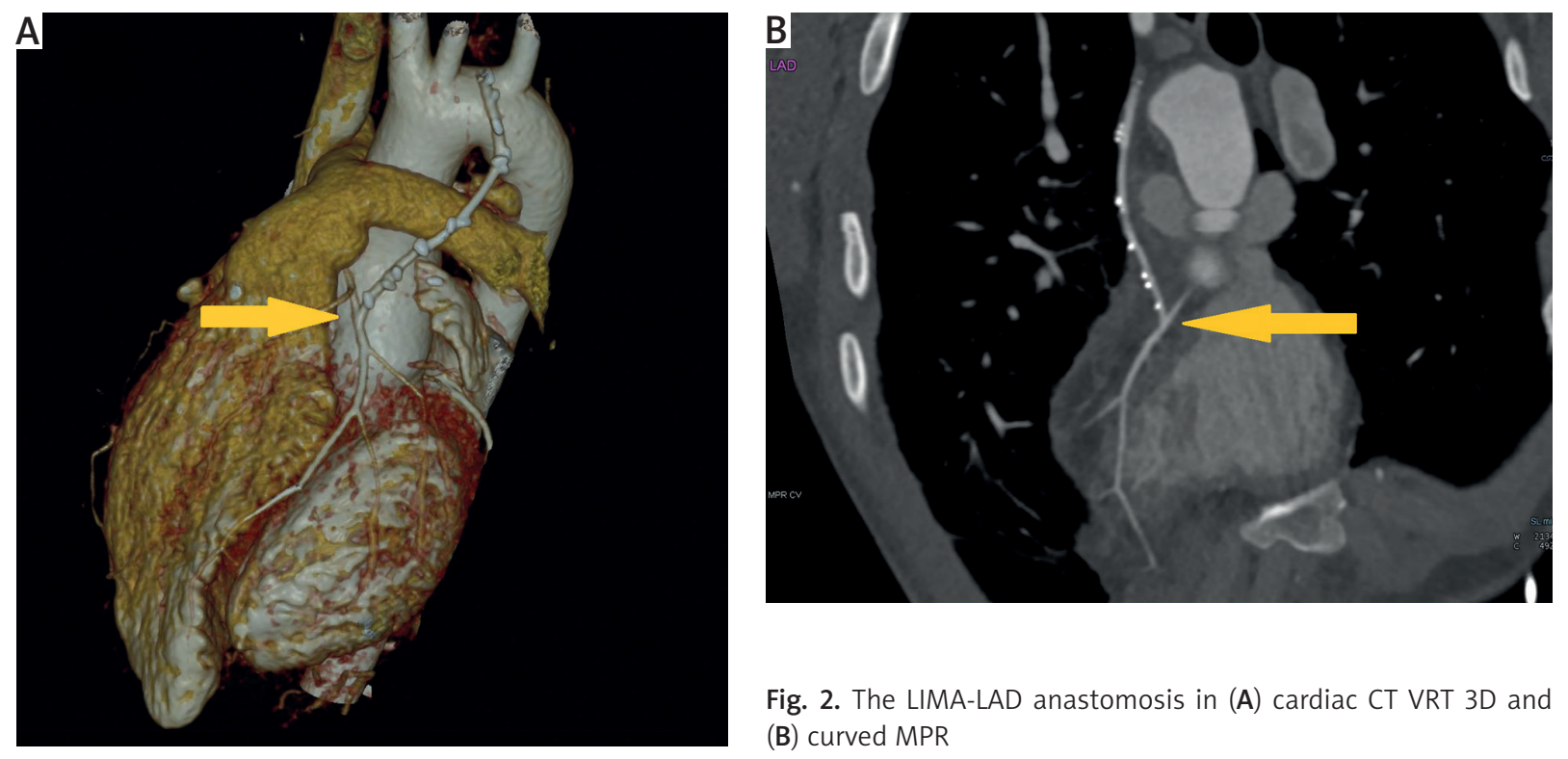

Fig. 2. The LIMA-LAD anastomosis in (A) cardiac CT VRT 3D and (B) curved MPR 
Coronary lesions are not rare complications in the follow-up after arterial switch operation. Some treatment options such as percutaneous interventions, surgical reconstruction or coronary artery bypass grafting are discussed $[7,8]$.

In our opinion performing surgical reconstruction would be a technically complex operation. There would be a need of intervention on both the neo-aorta and pulmonary trunk. On the other hand, percutaneous coronary interventions in the $L A D$ are known to have a worse clinical outcome than LIMA-LAD bypass [8].

This is the second available case report on performing MIDCAB after an arterial switch operation [9]. There are some cases of off-pump coronary bypass grafting via full sternotomy in these patients [10]. Our center performs both MIDCAB and off-pump coronary artery surgery as a routine.

Minimally invasive direct coronary artery bypass grafting is a minimally invasive surgical approach for single LAD disease. Reported studies showed high LIMA graft patency and low incidence of wound complications in the followup. In this particular case, avoiding repeated sternotomy resulted in prompt postoperative recovery.

On the other hand, this case showed how challenging this patient' $s$ cohort can be. The complex coronary situation may lead to occurrence of ventricular fibrillation. Quick use of femoral extracorporeal circulation maintained the safety for the patient and the procedure was successfully finished without any drawbacks for the patient. Consequently, as the procedure is technically more demanding it should be performed in centers experienced in both coronary and congenital heart defect surgery. Additionally, we decided to perform the CT scan after the operation to ensure the quality of the anastomosis.

In conclusion, we suggest that MIDCAB is an attractive revascularization option for patients after an arterial switch operation when performed in experienced hands.
Treatment of late coronary complications after an arterial switch operation should be performed in experienced centers performing both congenital and coronary surgery. The complex anatomy situation may lead to challenging situations during the procedure.

\section{Disclosure}

The authors report no conflict of interest.

\section{References}

1. Martins P, Castela E. Transposition of the great arteries. Orphanet J Rare Dis 2008; 3: 27.

2. Jatene AD, Fontes VF, Paulista PP, de Souza LC, Neger F, Galantier M, Sousa JE. Successful anatomic correction of transposition of the great vessels. A preliminary report. Arq Bras Cardiol 1975; 28: 461-464.

3. Fricke TA, d'Udekem Y, Richardson M, Thuys C, Dronavalli M, Ramsay JM, Wheaton G, Grigg LE, Brizard CP, Konstantinov IE. Outcomes of the arterial switch operation for transposition of the great arteries: 25 years of experience. Ann Thorac Surg 2012; 94: 139-145

4. Tsuda T, Bhat AM, Robinson BW, Baffa JM, Radtke W. Coronary artery problems late after arterial switch operation for transposition of the great arteries. Circ J 2015; 79: 2372-2379.

5. van Wijk SWH, van der Stelt F, Ter Heide H, Schoof PH, Doevendans PAFM, Meijboom FJ, Breur JMPJ. Sudden death due to coronary artery lesions longterm after the arterial switch operation: a systematic review. Can J Cardiol 2017; 33: 1180-1187.

6. Cisowski M, Morawski W, Drzewiecki J, Kruczak W, Toczek K, Bis J, Bochenek $A$. Integrated minimally invasive direct coronary artery bypass grafting and angioplasty for coronary artery revascularization. Eur J Cardiothorac Surg 2002; 22: 261-265.

7. Raisky O, Bergoend E, Agnoletti G, Ou P, Bonnet D, Sidi D, Vouhe PR. Late coronary artery lesions after neonatal arterial switch operation: results of surgical coronary revascularization. Eur J Cardiothorac Surg 2007; 31: 894-898.

8. El-Segaier M, Lundin A, Hochbergs P, Jögi P, Pesonen E. Late coronary complications after arterial switch operation and their treatment. Catheter Cardiovasc Interv 2010; 76: 1027-1032.

9. Poncelet AJ, Moniotte S, Momeni M, Detaille T, Rubay JE. Off-pump revascularization in a 2-year-old girl after neonatal arterial switch. Ann Thorac Surg 2014; 98: 1454-1456.

10. Jussli-Melchers J, Haneya A, Hoffmann G, Cremer J. Minimally invasive direct coronary artery bypass in a child with an occlusion of left main coronary artery after arterial switch operation. Interact Cardiovasc Thorac Surg 2013; 17: 1040-1041. 